Revue

Revue de l'histoire des religions

de Ihistoire des religions

1 | 2016

Varia

\title{
« Le ciel est partout » : l'espace spirituel dans la pensée de Jacob Boehme
}

"Heaven is everywhere": The spiritual space in Boehme's thought

\section{Mariel Mazzocco}

\section{OpenEdition}

\section{Journals}

Édition électronique

URL : http://journals.openedition.org/rhr/8481

DOI : $10.4000 /$ rhr.8481

ISSN : 2105-2573

\section{Éditeur}

Armand Colin

Édition imprimée

Date de publication : 1 mars 2016

Pagination : 5-19

ISBN : 978-2-200-93059-2

ISSN : 0035-1423

Référence électronique

Mariel Mazzocco, « « Le ciel est partout » : l'espace spirituel dans la pensée de Jacob Boehme », Revue de l'histoire des religions [En ligne], 1 | 2016, mis en ligne le 01 mars 2019, consulté le 04 septembre 2020. URL : http://journals.openedition.org/rhr/8481 ; DOI : https://doi.org/10.4000/rhr.8481 
MARIEL MAZZOCCO

Université de Genève

\section{«Le ciel est partout» : l'espace spirituel dans la pensée de Jacob Boehme}

Considéré par Alexandre Koyré comme l'un des penseurs les plus énigmatiques de l'univers, Jacob Boehme - dénommé le Philosophus Teutonicus - soulève encore aujourd'hui bien des questions et constitue un défi interprétatif. Cet article se propose d'analyser la notion d'espace dans les écrits mystiques de Boehme et de retracer la topographie spirituelle de l'âme perdue dans les chemins invisibles du ciel ou égarée dans les ténèbres.

\section{"Heaven is everywhere": The spiritual space in Boehme's thought \\ Considered by Alexandre Koyré as one of the most enigmatic thinkers of the universe, Jacob Boehme - called the Philosophus Teutonicus - still today raises many questions and he represents an intellectual challenge. This article aims to analyze the notion of space in Boehme's mystical writings and to trace the soul's topography lost in the invisibles ways of the heaven or in the darkness.}




\section{LA RÉCEPTION D'UN PENSEUR ÉNIGMATIQUE}

En 1800, dans sa préface à la première édition française de l'Aurora de Jacob Boehme, Louis Claude de Saint-Martin avouait que la doctrine du théosophe allemand «pénètre dans des régions où nos langues manquent si souvent de mots pour s'exprimer ${ }^{1}$.

C'est durant un long séjour à Strasbourg vers la fin du XVIII e siècle que Saint-Martin, dit le Philosophe Inconnu, avait découvert l'œuvre du Philosophus Teutonicus. Séduit par la dimension métaphysique de Boehme, Saint-Martin se lança dans une entreprise à la fois compliquée et délicate: traduire les livres du cordonnier mystique de Görlitz. Il se consacra à cette tâche avec un tel enthousiasme qu'il dut interrompre momentanément la rédaction de ses propres ouvrages pour se dédier exclusivement au travail de traduction ${ }^{2}$. L'auteur de L'homme de désir et de Ecce homo trouva dans les écrits de Boehme des convergences avec sa propre pensée, notamment au sujet de l'accès à la connaissance divine. Plongé dans ces écrits mystiques, le fondateur du Martinisme vit une saison spirituelle très féconde et entre dans la «paix de la régénération», qui était, d'après le penseur allemand, indispensable pour atteindre la Divinité. «Malheur à celui qui ne fonde pas son édifice spirituel sur la base solide de son cœur en perpétuelle purification et immolation par le feu sacré, écrivait Saint-Martin dans son Portrait historique et philosophique ${ }^{3}$, c'est à mon incomparable Boehme que je dois d'avoir fait cette réflexion sur moi-même».

Saint-Martin prit ainsi la décision de rendre accessible au public français les puissantes thèses métaphysiques du théosophe allemand.

1. Jacob Boehme, L'aurore naissante, ou la racine de la philosophie, de l'astrologie et de la théologie, traduite par Louis-Claude de Saint-Martin, Paris, Imprimerie de Laran, 2 vol., 1800, avertissement du traducteur (nous ferons ici référence à la réimpression publiée à Milan, chez Archè, collection Sebastiani, en 1977).

2. Saint-Martin s'était servi de l'édition allemande des œuvres de Boehme parue en 1682 à Amsterdam. Une autre édition des œuvres complètes de Boehme avait paru en 1715, puis en 1730, sous le titre Theosophia Revelata. Une réimpression en fac-similé de l'édition de 1730 a été publiée sous le titre Jacob Böhme: Sämtliche Schriften (11 vol.) à Stuttgart en 1955-1961.

3. Louis-Claude de Saint-Martin, Mon portrait historique et philosophique (1789-1803), 427, Paris, R. Julliard, 1961, p. 221. 
Dans l'espace de quatre ans il avait traduit quatre ouvrages. Deux seront publiés du vivant de Saint-Martin («L'Aurore naissante» en 1800 et «Des trois principes de l'essence divine» en 1802), les deux autres à titre posthume ("Quarante questions sur l'origine, l'essence, l'être, la nature et la propriété de l'âme» en 1807 et «De la triple vie de l'homme» en 1809). Par l'intermédiaire de Saint-Martin les œuvres de Boehme commencèrent à circuler en France, en alimentant maintes générations d'intellectuels en quête de l'insaisissable, entre autres Honoré de Balzac, qui, déjà influencé par Swedenborg, dans la bibliothèque de sa mère avait découvert les œuvres de Saint-Martin ainsi que sa traduction de Boehme ${ }^{4}$.

Il faut donc reconnaître l'immense mérite de Saint-Martin concernant la diffusion de la pensée du cordonnier de Görlitz en France, d'autant plus qu'au fil des siècles personne n'a plus eu le courage d'entreprendre une nouvelle traduction française de l'Aurora. Nous devons d'ailleurs admettre que la langue de Boehme est particulièrement difficile; Saint-Martin lui-même avouait avoir choisi de «faire une traduction exacte et fidèle, plutôt qu'une traduction élégante $»^{5}$. En outre Boehme était bien connu pour ses créations linguistiques, difficilement traduisibles dans une autre langue. Il suffit d'évoquer des termes tels que Salitter, Turba, Urkund, qui occupaient une place majeure dans l'architecture cosmologique boehmienne.

Si traduire les œuvres de Jacob Boehme s'avère délicat, l'interprétation de sa doctrine soulève plusieurs questions, souvent difficiles à résoudre d'une manière univoque. Boehme ne pense pas par notions distinctes mais par antithèses et symboles. Sa pensée ne se fige pas, elle est en mouvement, toujours in fieri. Conscient de toutes ces difficultés, en 1802, dans le Ministère de l'homme-esprit, Saint-Martin se sentait en devoir de mettre en garde le lecteur:

Je croirai rendre un service au lecteur en l'engageant à faire connaissance avec cet Auteur; mais en l'invitant surtout à s'armer de patience et de courage pour n'être pas rebuté par la forme peu régulière de ses ouvrages, par l'extrême abstraction des matières qu'il traite, et par la difficulté qu'il avoue lui-même avoir eue à rendre ses idées, puisque la plupart des matières en question n'ont point de noms

4. À ce propos, voir par exemple P. Laubriet, L'intelligence de l'Art chez Balzac, Genève, Slatkine, 1980, en particulier le chapitre «La postulation mystique», p. 286-318.

5. L'Aurore naissante, p. 17. 
analogues dans nos langues connues. [...] Lecteur, si tu te détermines à puiser courageusement dans les ouvrages de cet auteur, qui n'est jugé par les savants dans l'ordre humain, que comme un épileptique, tu n'auras sûrement pas besoin des miens ${ }^{6}$.

Abstraits et paradoxaux, ces ouvrages se prêtent à des interprétations divergentes. Considéré à la fois comme «le premier philosophe allemand $»^{7}$ et un grand philosophe chrétien ${ }^{8}$, un idéaliste et un panthéiste, Boehme demeure encore aujourd'hui l' «un des penseurs les plus énigmatiques de l'univers ${ }^{9}$.

Comme l'avait bien saisi Émile Boutroux, l'œuvre de Boehme est «un mélange confus de théologie abstruse, d'alchimie, de spéculations sur l'insaisissable et l'incompréhensible, de poésie fantastique et d'effusions mystiques: c'est un chaos étincelant ${ }^{10}$. D’ailleurs déjà Balzac avouait aimer à se plonger dans ce «monde mystérieux, invisible aux sens où chacun se plaît à vivre, soit qu'il se le représente sous la forme indéfinie de l'avenir, soit qu'il le revête des puissantes formes de la Fable ${ }^{11}$. En outre, comme le remarquait Koyré, étudier Boehme est particulièrement difficile car «chacune de ses œuvres est une exposition complète de tout son système; et les redites sont aussi fréquentes que les contradictions $\gg^{12}$.

6. Louis-Claude de Saint-Martin, Le ministère de l'homme-esprit, Paris, Migneret, 1802, p. 29-31. Voir aussi la lettre envoyée au baron Kirchberger en 1792: «C'est avec franchise, monsieur, que je reconnais n'être pas digne de dénouer les cordons des souliers de cet homme étonnant, que je regarde comme la plus grande lumière qui ait paru sur la terre après Celui qui est la lumière même » (La Correspondance inédite de L. C. de Saint-Martin dit le Philosophe Inconnu et Kirchberger, baron de Liebistorf, Ouvrage recueilli et publié par L. Schauer et A. Chuquet, Paris, E. Dentu, 1862, Lettre du 8 février 1792, p. 9).

7. Voir G. W. F. Hegel, Vorlesungen über die Geschichte der Philosophie, in Werke, éd. E. Moldenhauer et K. M. Michel, 20 voll., Frankfurt am Main, Suhrkamp Verlag, 1971-1981, vol. XX, p. 94; trad. fr. P. Garniron, Leçons sur l'histoire de la philosophie, Paris, Vrin, coll. «Bibliothèque des textes philosophiques », 1985, t. VI, p. 1304. Rappelons que Hegel possédait un exemplaire de l'édition des œuvres complètes de Boehme publiée à Hambourg en 1715.

8. Cf. Franz von Baader, Vorlesungen über Jacob Böhmes Theologumena und Philosophema, in Sämtliche Werke, t. III, Leipzig, 1852, p. 357 suiv.

9. Alexandre Koyré, La philosophie de Jacob Boehme, Paris, Vrin, 1971 (2e éd.), p. XV.

10. Emile Boutroux, Le philosophe allemand Jacob Boehme, Paris, F. Alcan éditeur, 1888, p. 5.

11. Honoré de Balzac, Histoire intellectuelle de Louis Lambert (1832), XXXI, 81.

12. La philosophie de Jacob Boehme, p. 11. 
Pour tenter d'éclairer l'énigmatique pensée du Philosophus teutonicus il faudrait plutôt s'insérer dans le sillage des écrivains mystiques (notamment Angelus Silesius et Emmanuel Swedenborg, ainsi que Saint-Martin) qui avaient su déceler son message secret. Ce message, caché dans les replis clairs obscurs d'une écriture souvent difficile et d'une doctrine parfois contradictoire, consiste dans le thème de la régénération (Wiedergeburt) de l'homme qui est au cœur de l'anthroposophie de Boehme. Par rapport à une cosmologie et une théologie confuses, dont le langage d'inspiration paracelsiste dérange le lecteur moderne, sa réflexion anthropologique anime la quête identitaire de l'homme déchiré entre le bien et le mal. Écriture d'un non-lieu, l'œuvre de Boehme ne saurait que l'esquisse d'une topographie de l'âme perdue dans les chemins invisibles du ciel ou égarée dans les ténèbres.

Laissons donc en arrière-plan le spectacle de l'univers peint par le théosophe allemand, et assistons à celui plus intime qui se consomme sur la scène de l'esprit, au fond du cœur.

\section{LUMIÈRES ET TÉNÈBRES: DEUX MONDES INCONCILIABLES?}

La question de l'origine du mal dans l'œuvre de Boehme est très compliquée, voire confuse. S'il privilégie la thèse de la chute originelle provoquée par la révolte de l'ange rebelle brisant l'ordre divin ${ }^{13}$ (à ses yeux, la prévarication d'Adam est donc moins grave), Boehme soutient également que le mal est coexistant à Dieu. Dans la sagesse divine, lumières et ténèbres, amour et colère cohabitent, car Dieu «a introduit ensemble le feu et la lumière, le bien et le mal dans le Fiat, dans une libre volonté où la volonté peut se former dans le Bien ou le Mal $»^{14}$. Contrairement à ses prédécesseurs, Valentin Weigel et Sébastien Franck, qui, s'inscrivant dans la tradition augustinienne du mal

13. Voir L'Aurore naissante, XVI, 26, p. 269: «Quand tous les arbres seraient des écrivains; toutes les branches, des plumes; toutes les montagnes, des livres; et toutes les eaux, de l'encre, ils ne pourraient pas encore suffire pour décrire la misère et les souffrances que Lucifer avec ses anges a apportées dans la place qu'il occupait».

14. J. Boehme, Mysterium Magnum (1623), trad. fr. S. Jankélévitch, Paris, Aubier, 1945, ch. 28, p. 335. 
comme privation du bien ${ }^{15}$, avaient insisté sur son inconsistance ontologique, Boehme conçoit le mal comme une entité surgie de l'Imagination créatrice divine. Alors qu'au début du XVII siècle, notamment en France avec le courant bérullien, on définissait le péché comme un néant ${ }^{16}$, Boehme l'inscrit dans la région de l'être. Dans la mesure où Dieu comprend toute chose, le bien et le mal ne sont pas deux mondes inconciliables, au contraire, l'un ne peut être pensé sans l'autre. Comme le remarquait Hegel, Boehme s'efforce de maintenir toutes les choses dans une unité supérieure: "l'unité divine absolue et l'unification de tous les opposés en Dieu $»^{17}$. L'opposition bien-mal ne suppose pas une séparation ontologique, ce sont plutôt les deux aspects différents mais complémentaires d'une même réalité en mouvement. Sous la plume du cordonnier mystique, la lumière surgit des ténèbres, le mal manifeste le bien:

La force dans la lumière est le feu d'amour de Dieu et la force dans les ténèbres est le feu de l'ire divine et pourtant il ne s'agit que d'un seul feu. Mais il se scinde en deux principes, afin que l'un se manifeste en l'autre. Car la flamme de la colère est la révélation du grand amour; c'est dans les ténèbres qu'on connaît la lumière, sinon elle ne se manifesterait pas ${ }^{18}$.

On a considéré Boehme comme «l'un des plus grands gnostiques chrétiens ${ }^{19}$. La question certes est complexe, pourtant dans l'œuvre du théosophe allemand il n'y a pas un véritable dualisme ontologique. C'est en tant que principe absolu et origine première de toutes choses que Dieu comprend le mal. «Source de colère et d'amour» Dieu a tout créé «dans une essence créaturelle et figurative, bonne et mauvaise, selon l'éternelle source ${ }^{20}$. Ce n'est

15. Saint Augustin, De natura boni, I, 17: «Non ergo mala est, in quantum natura est, ulla natura; sed cuique naturae non est malum nisi minui bono.» Voir aussi la définition du mal comme «corruptio vel modi, vel speciei, vel ordinis naturalis».

16. Voir Pierre de Bérulle, Euvres de piété, n 227, Euvres complètes, vol. IV, Paris, Cerf, 1996, p. 140.

17. Hegel, Leçons sur l'histoire de la philosophie, p. 1310.

18. Mysterium Magnum, ch. 8, p. 102.

19. N. Berdiaeff, L'Ungrund et la liberté, in Mysterium Magnum, p. 5. Au sujet des éventuelles traces de manichéisme et de gnosticisme dans la doctrine de Boehme, cf. F. Ch. Baur, Die christliche Gnosis, Tübingen, 1835, p. 586 suiv. et A. Stöckl, Geschichte der Philosophie des Mittelalters, III, Mainz, 1866, p. 583 et suiv.

20. J. Boehme, Sex puncta theosophica (1620); trad. fr. de L.-C. de Saint-Martin, De la base sublime et profonde des six points, Paris, Migneret, 1807, I, 2, p. 331. 
que virtuellement, et non de manière active, que Dieu contient le mal. Dans son bouillonnement, l'essence divine «imagine» le mal, sans l'incarner. Le mal est un monde possible que seule la volonté créée peut choisir d'habiter. Boehme saisit très bien la misère et la grandeur de la volonté humaine ayant le pouvoir de déformer et noircir l'âme ou de la transformer en un ange de lumière:

La volonté peut donner à l'esprit même une autre forme, ainsi qu'il suit: quand même l'esprit serait un ange, une image de Dieu, la volonté peut néanmoins faire de lui un démon insensé, de même que d'un démon faire un ange, pourvu qu'il se précipite dans la mort, dans l'humilité sous la croix, et qu'il se plonge de nouveau dans l'esprit de Dieu, en sorte qu'il se soumette à son gouvernement ${ }^{21}$.

En lisant ce passage on ne peut ne pas songer à Pierre de Bérulle, qui, à la même époque, dans ses opuscules de piété écrivait que l'homme «est céleste d'une part et terrestre de l'autre. Il est spirituel d'une part et corporel de l'autre. C'est un ange, c'est un animal; c'est un néant, c'est un miracle $»^{22}$. Plus connue est la célèbre pensée de Pascal, qui dans des termes semblables (il connaissait les œuvres complètes de Bérulle éditées par Bourgoing en 1644) chantait l'homme «ni ange ni bête $»^{23}$.

D'après Boehme «la vie humaine est l'angle entre la lumière et la ténèbre; celui auquel elle se donne, est celui dans lequel elle brûle $»^{24}$. Si l'âme demeure dans l'extérieur, elle est dans «l'enfer». Il faut donc regarder ailleurs, se réfugier dans les recoins secrets du cœur, car le paradis se cache au fond de l'âme. L'âme peut donc choisir où aller, si entrer en elle-même et trouver le paradis, ou sortir à l'extérieur et habiter l'enfer. Mais comment trouver le Paradis sans s'égarer dans le labyrinthe obscur du mal? Influencé par Paracelse ${ }^{25}$, le théosophe

21. J. Boehme, Psychologia vera (1620); trad. fr. de L.-C. de Saint-Martin, Quarante questions sur l'origine, l'essence, l'être, la nature et la propriété de l'âme, Paris, Migneret, 1807, VI, 14, p. 111.

22. P. de Bérulle, CEuvres de piété, n ${ }^{\circ} 168$, p. 10.

23. Blaise Pascal, Pensées, pansée 358, éd. Léon Brunschvicg, in Euvres, vol. XIII, Paris, Hachette, 1904. Cf. aussi pensée 418: «Il ne faut pas que l'homme croie qu'il est égal aux bêtes, ni aux anges, ni qu'il ignore l'un et l'autre, mais qu'il sache l'un et l'autre».

24. Courte explication des six points, in De la base sublime et profonde des six points, p. 443.

25. Voir en particulier Paracelse, Philosophia sagax, livre II, § 2, in Euvres, éd. Huser, Bâle, 1589, vol. X, p. 263 et De generatione stultorum, in Euvres, vol. IX, p. 29. 
allemand soutient que l'âme, en tant qu'«image de l'universel», est un microcosme et un «microtheos» qui englobe l'univers et Dieu. Dans l'œuvre de Boehme tout est un jeu de reflets et de miroirs, car «tous les êtres sont magiques» (alle wesen magisch sind), et «l'un est le miroir de l'autre ${ }^{26}$. Enfer et Paradis ne se trouvent pas ailleurs, ils sont coextensifs à l'espace, immanents à l'univers intérieur ainsi qu'au monde extérieur qui scelle les vestiges d'un Dieu présent en tout.

La notion d'espace est très importante dans l'œuvre du théosophe de Görlitz. En 1612, dans l'Aurora, son premier traité «conçu dans un esprit presque magique $»^{27}$, Boehme avouait que personne ne peut savoir combien est «étendu et profond le lieu de ce monde», et il ajoutait que «le vrai ciel est par-tout, dans ce temps actuel» ainsi que «la maison de la colère de l'enfer $»^{28}$. En 1619 il revient sur cette thèse dans le traité De Tribus Principiis, précisant que «le vrai ciel où Dieu demeure est partout en tout lieu, ainsi qu'au milieu de la terre. Il comprend l'enfer où le démon demeure, et il n'y a rien hors de Dieu ${ }^{29}$. Une année plus tard, en 1620, probablement inspiré par les thèses Weigeliennes ${ }^{30}$, Boehme écrit encore que «chaque âme est dans sa propre contrée [lande], et n'est point liée à la place [Stelle] qu'occupe le corps; mais elle peut aller où elle veut. Quelque part où elle soit, elle est ou dans Dieu, ou dans les ténèbres $»^{31}$.

Il ne faut donc pas aller loin pour trouver le Paradis:

Si vous voulez considérer ce qu'est le ciel, où il est, ou bien comment il est; vous n'avez pas besoin d'élancer votre pensée à plusieurs milliers de milles d'ici. Car cet espace ou ce ciel n'est pas votre ciel [...] Car le vrai ciel est par-tout, même dans le lieu où vous êtes et où vous marchez. Lorsque votre esprit atteint la génération la

26. Quarante questions, 18, 3, p. 165.

27. Voir J. Boehme, Epistolae theosophicae (1618-1624), XVIII, 13; trad. fr., de B. Gorceix, Les Épîtres théosophiques, Paris, Éditions du Rocher, 1980.

28. L'Aurore naissante, XXV, 19-20, p. 422.

29. J. Boehme, De Tribus Principiis (1619): «Der rechte Himmel, da Gott innen wohnet, ist überall an allen Orten, auch mitten in der Erden: Er begreift die Hölle, da die Teufel wohnen, und ist nichts ausser Gott»; trad. fr. L.-C. de SaintMartin, Des trois Principes de l'essence divine, ou de l'éternel engendrement sans origine, Paris, Imprimerie de Laran, 1802, p. 94.

30. Voir Valentin Weigel, Vom Ort der Welt (Hall in Sachsen, 1613) chap. X et XIV, où Valentin Weigel développe sa notion d'espace qui pourrait être résumée en ces mots: omnis locus est in mundo et mundus non est in loco.

31. Quarante questions, XXI, 20, p. 181. 
plus intérieure de Dieu, et qu'il pénètre au travers de la génération sidérique et charnelle, dès-lors il est dans le $\operatorname{ciel}^{32}$.

En lisant ce beau passage de l'Aurora on songe immédiatement au célèbre distique de Silesius :

I,82. Le ciel est en toi.

Arrête, où cours-tu donc, le ciel est en toi :

et chercher Dieu ailleurs, c'est le manquer toujours ${ }^{33}$.

Par ces mots, et bien d'autres éparpillés dans le Cherubinischer Wandersmann, l'on mesure l'influence de Boehme sur Silesius ${ }^{34}$. On sait que le mystique silesien avait commencé à lire l'œuvre du Philosophus Teutonicus durant son séjour en Hollande et cette influence s'était exercée encore à Oels, où Frankenberg, le biographe de Boehme, lui avait parlé de son maître. Sous la plume $\mathrm{du}$ poète baroque allemand nous retrouvons quelques-unes des idées caractérisant la pensée spirituelle de Boehme ${ }^{35}$. Pour tous les deux «le lieu est Dieu $»^{36}$ et cet espace lui-même se trouve dans l'âme. Nul besoin alors de chercher le ciel: «Il est dans notre cœur. Ouvrez-le seulement: la clef vous en est montrée ici» ${ }^{37}$.

32. L'Aurore naissante, p. 315 (Aurora, XIX, 23-24: «Wenn du deine Gedanken von dem Himmel fassest, was der sei oder wo der sei, so darfst du deine Gedanken nicht viel tausend Meilen von Hinnen schwingen, denn derselbe Locus oder Himmel ist nicht dein Himmel [...]Denn der rechte Himmel ist allenthalben, auch an dem Orte, wo du stehest und gehest. Wenn dein Geist die innerste Geburt Gottes ergreift und durch die Siderische und fleischliche Hindurchdringet, So ist er schon im Himmel»). Cf. aussi plus loin, XXIII, 9, p. 390: «Lorsque vous considérez l'espace, les étoiles et la terre, alors vous voyez votre Dieu; vous vivez, et vous êtes dans ce même Dieu, et ce même Dieu vous gouverne aussi, et c'est de ce même Dieu que vous tenez également vos pensées ».

33. Angelus Silesius, Pèlerin chérubinique (Cherubinischer Wandersmann), trad. fr. de H. Plard, Paris, Aubier, 1946, p. 74-75: «Der Himmel ist in dir. Halt an wo lauffstu hin, der Himmel ist in dir: / Suchstu Gott anderswo, Du fehlst Ihn für und für».

34. À ce propos nous renvoyons à Jean Orcibal, La Formation spirituelle d'Angelus Silesius (1624-1677). Mystique et Contre-Réforme, Mémoire de l'École Pratique des Hautes Études (exemplaires dactylographiées), Paris, 1937.

35. En ce qui concerne le thème de la transmutation spirituelle d'inspiration alchimiste, voir notamment les distiques I, 202-203; I, 244; III, 118. Au sujet des images musicales, je me permets de renvoyer à mon étude «Entre silence et vibrations sonores: la poésie mystique d'Angelus Silesius », in Rivista di Storia e Letteratura Religiosa, Firenze, Olschki, vol. 49, n 2, 2013, p. 443-456.

36. Pèlerin chérubinique, I, 205, p. 92-93: «Der Ort ist dass Wort. Der ort und's Wort ist Eins, und wäre nicht der ort, / (Bey Ewger Ewigkeit!) es wäre nicht dass Wort»; «Le Lieu est Dieu. Le lieu et Dieu sont un; s'il n'y avait le Lieu (par toute étérnité !) il n'y aurait pas Dieu».

37. L'Aurore naissante, XXIII, 71, p. 400. 


\section{UNE RENAISSANCE MAGIQUE}

Pourtant Boehme est contraint de reconnaître que la plupart des gens ne trouvent pas ce chemin vers le ciel et préfèrent se confondre dans la turba. D'après lui pour habiter les régions de la Divinité il faut s'ouvrir à Dieu, choisir une autre vie et se transformer. Comment atteindre cette régénération? Et, surtout, pourquoi estelle indispensable aux yeux du luthérien Jacob Boehme alors que selon la position de Martin Luther par son sacrifice sanglant le Christ avait obtenu le pardon des péchés ? Böhme ne veut sans doute pas nier l'efficacité de la justification : l'homme ne suffit pas à soi et ne peut pas se passer de la Grâce, mais pour s'avérer efficace la Grâce ne doit pas être acceptée passivement, mais activement. Si l'œuvre du salut n'est plus à attendre, néanmoins pour renaître en Jésus-Christ il faut que l'homme s'ouvre à l'Autre et se libère de la pesanteur du «moi » afin de laisser à l'Esprit la possibilité de le régénérer.

Ainsi par ses écrits le cordonnier mystique souhaitait allumer «la faim de l'âme avide de naître de nouveau ${ }^{38}$. Il s'agit de mourir à soi pour renaître dans le Christ. Cette renaissance est souvent décrite par le biais d'images empruntées à la doctrine alchimiste. La régénération de l'âme est assimilée à la transmutation des métaux. Tel un «mage alchimiste ${ }^{39}$, l'homme doit faire en sorte que l'élément divin se répande dans l'organisme spirituel: l'âme n'étant qu'un «feu magique ${ }^{40}$, elle peut brûler et se changer en or. S'exprimant en termes d'alchimie spirituelle, le théosophe conçoit la transformation intérieure de l'âme comme relevant d'une opération quasi magique. Le mot Magie en effet occupe un rôle important dans l'œuvre de Boehme. Mais qu'est-ce que la magie? «La vraie magie n'est aucun être - explique-t-il - mais l'esprit désirant l'être ${ }^{41}$.

Selon Boehme la puissance magique par excellence est l'imagination (Einbildung), car elle peut transformer l'âme dans l'image qu'elle imite. Koyré écrivait que l'Imaginatio est une puissance

38. J. Boehme, De signatura rerum (1622), De la signature des choses, trad. fr. P. Deghaye, Paris, Grasset, 1995, XI, 69, p. 230.

39. De la signature des choses, VII, ch., p. 124.

40. Courte explication des six points, p. 440.

41. Ibid., p. 454. 
plastique qui coule l'homme dans la forme imaginée par lui ${ }^{42}$. Dans l'imitatio Christi l'homme incarne en lui l'image de la Divinité, et dans cette image (vorbild) désirée, il reconstruit sa propre identité. On dira alors, d'après la formule de Boehme, que «tout naît par l'imagination et le désir $»^{43}$.

Le processus de régénération est une opération constructive, transformante, et non destructive ou annihilante. Bien qu'il reprenne le thème de la mort spirituelle, la Gelassenheit, cher à la mystique allemande, Boehme ne conçoit pas la renaissance de l'âme en termes d'anéantissement. D'une certaine manière il rompt avec la tradition rhéno-flamande qui, s'inspirant de l'adage augustinien Funde ut implearis ${ }^{44}$ invitait l'homme à s'anéantir pour être rempli de l'essence divine. C'est le cas notamment de Tauler, que Boehme connaissait par l'intermédiaire de Weigel: dans ses sermons il avait montré quel degré d' «anéantissement illimité » ${ }^{45}$ il faillait atteindre pour être possédé par la Divinité.

À la même époque de Boehme, le cardinal de Bérulle, inspiré par la lecture de la Perle Évangélique, abordait le thème délicat du «néant capable de Dieu $»^{46}$. Alors qu'en France, au début du XVII ${ }^{\mathrm{e}}$ siècle, «l'invasion mystique ${ }^{47}$ avait décrété le succès du langage abstrait nihiliste, à Görlitz le cordonnier mystique faisait preuve d'originalité. Si l'âme se «donne dans un rien (ou l'abnégation absolue), écrivait-il en 1620, alors elle est dénuée de désir ${ }^{48}$. Ce rejet du thème de l'anéantissement a des enjeux importants. Si les mystiques du Nord se plaisaient à invoquer l'abîme

42. Cf. La philosophie de Jacob Boehme, p. 481-482.

43. De la signature des choses, VIII, 11, p. 146.

44. Augustin, Enarrationes in Psalmos, In Psalmo 30, Sermo 3, chap. 11, PL 36, col. 254.

45. J. Tauler, Sermons, Paris, Éd. du Cerf, 1991, p. 71.

46. Euvres de piété, $\mathrm{n}^{\circ} 168$, p. 10. Voir aussi La Perle évangélique (1602), éd. D. Vidal, Grenoble, J. Millon, 1997, p. 292: «Là alors sera l'essence comprise de l'essence. Là ce rien, c'est-à-dire Dieu, est rencontré de cet autre rien, c'est-à-dire de l'âme. Là rien, qui est cette âme, est enveloppée et noyée dedans le rien, c'està-dire Dieu. Là enfin le rien est absorbé et englouti du rien.»

47. Selon la formule employée par l'abbé Bremond dans son Histoire littéraire du sentiment religieux en France (Paris, Bloud et Gay, 1921, nouvelle éd. sous la direction de F. Trémolières, Grenoble, Jérôme Millon, 2006; voir le tome intitulé «L'invasion mystique»). Sur ce thème, voir aussi Sophie Houdard, Les Invasions mystiques. Spiritualités, hétérodoxies et censures au début de l'époque moderne, Paris, Les Belles Lettres, 2008.

48. Courte explication des six points, p. 443. 
sans fond ${ }^{49}$ (abgrund), Boehme préfère chercher un Dieu vivant se répandant dans le monde. Pendant les sept années de silence où on lui avait interdit d'écrire suite à la condamnation de l'Aurora le 30 juillet 1613, il avait plongé dans la lecture de Paracelse, Kaspar Schwenkfeld, Sébastian Franck et Valentin Weigel ${ }^{50}$.

Par l'intermédiaire de Franck et Weigel, c'est avec les grands mystiques rhéno-flamands (Eckhart, Tauler, Ruusbroec) qu'il était entré en contact. Pourtant si ces lectures le marquent, c'est pour s'en distinguer. Certes, il puise dans le langage de la mystique abstraite, mais il réinterprète le sens des mots et des notions à sa façon. S'inscrivant dans la tradition apophatique du Pseudo-Denys, Maître Eckhart et ses disciples (Tauler et Suso) avaient considéré le «Néant surressentiel $»^{51}$ (überseiende Nichtheit) la sublime expression de la Divinité. C'est justement cette primauté du Rien sur l'Être que Boehme conteste.

Si pour le Maître Thuringien «ce néant était Dieu» ${ }^{52}$, pour le Philosophus Teutonicus l'abîme sans fond n'est qu'un germe de l'Absolu. Employé pour la première fois dans la Psychologia vera, le mot ungrund $d^{53}$ indique la source primitive, l'absolu, origine de toute chose. Cependant le sans-fond, qui est un éternel rien, ne peut pas être la meilleure expression de la Divinité, car il est caractérisé par une absence totale de détermination. Bref, le «Rien éternel» (ein ewig Nichts) ${ }^{54}$, indéterminé, aux yeux de Boehme n'est pas

49. Voir par exemple Tauler, Sermons, p. 356.

50. Sur la doctrine de ces auteurs nous renvoyons à A. Koyré, Mystiques, spirituels, alchimistes du XVII siècle allemand, Paris, Gallimard, 1971.

51. Maître Eckhart, Deutsche Predigten und Traktate, éd. J. Quint, Munich, Hanser Verlag, 1995, Predigt 42, p. 353; voir Sermons, trad. J. Ancelet-Hustache, Paris, Seuil, 1979, t. III, p. 152.

52. Idem, Sermons, vol. 3, Sermon 71, p. 75. Voir aussi Die Predigten Taulers, éd. Ferdinand Vetter, Berlin, Deutsche Texte des Mittelalters, vol. XI, 1910, [rééd. Dublin/Zurich, 1968], Predigt 32, p. 120. Voir aussi Henri Suso, Vie, dans Euvres complètes, éd. J. Ancelet-Hustache, Paris, Seuil, 1977, p. 303. Sur ce thème je me permets de renvoyer à mon étude: "Suressentiel. Aux sources d'un langage mystique», Revue de l'histoire des religions, 4, 2013, pp. 609-627.

53. Psychologia vera, I,16: «Also siehet der Ungrund in sich und findet sich selber» (voir Quarante questions, p. 7: «Ainsi le sans-fond voit en soi et se trouve luimême»). Au sujet des nuances entre ungrund et abgrund dans l'œuvre de Boehme, et sur l'emploi de ces mots par rapport à la tradition rhéno-flamande, voir par exemple l'étude de Virginie Pektaș, Mystique et philosophie: Grunt, abgrunt et Ungrund chez Maître Eckhart et Jacob Böhme, Amsterdam-Philadelphia, B. R. Grüner, 2006.

54. Mysterium Magnum, I, 2, vol. 1, p. 55. Sur ce «sans fond» qui est «un éternel rien», voir aussi Mysterium pansophicum (1620), trad. fr. de L.-C. de Saint Martin, Instruction fondamentale sur le mystère céleste et terrestre, in Quarante questions, p. 461. 
Dieu. Il ne possède aucune essence (wesen) et ne comporte ni lumière ni ténèbres: il est avant tout une «magie», écrit-il dans le De Incarnatione Verbi ${ }^{55}$.

La Divinité doit surgir de l'abîme. Le cordonnier mystique aborde cette question dans son ouvrage le plus systématique, Mysterium magnum, composé en 1623. «Le Néant, remarque Boehme, a faim du Quelque chose (Nichts hungert nach dem Etwas), et la faim est le désir, sous la forme du premier Verbum fiat ${ }^{56}$. Le Rien doit donc se fixer dans quelque chose. Cette dialectique du désir est le moteur d'un devenir divin. Boehme ne paralyse pas la Divinité dans le vide. Le Dieu de Boehme n'est pas détaché du monde, mais c'est un Dieu personnel caractérisé par un dynamisme secret. Dieu évolue intemporellement, Il est vivant, Il se développe dans l'infini ainsi que dans le monde qui nous environne. Le corps spirituel de Dieu est partout. L'espace est en Dieu et Dieu dans chaque point de l'espace. On a accusé Boehme de panthéisme, sans comprendre qu'en réalité pour le cordonnier de Görlitz Dieu est partout et nulle part, car il est immanent et transcendant à la fois l'univers.

Pourquoi rentrer dans le brouillard de l'inconnaissance alors que Dieu se manifeste dans le monde? Le monde est une expression de Dieu, les créatures, les animaux, la nature sont des Signatura où résonne le Verbe divin. Et si l'homme veut vraiment connaître Dieu en soi, dans son essence, Boehme donne ce conseil: «enlève la nature et la créature, ce qui reste est Dieu en soi $»^{57}$.

Le vide n'est pas le lieu de la rencontre entre Dieu et l'âme. Le refus d'un dieu Néant a des conséquences importantes sur l'anthropologie boehmienne. L'âme n'est pas appelée à s'anéantir pour se perdre dans l'abîme sans fond de la Divinité où les distinctions se raréfient jusqu'à disparaître. Il n'y a pas chez Boehme une mystique de la fusion en Dieu, ni une absorption identitaire dans le fond de l'âme. L'homme n'est pas invité à s'abandonner passivement à une volonté divine imperscrutable, mais à oser un changement radical

55. Von des Menschwerdung Jesu Christi (1620), II, 1-8.

56. Mysterium Magnum, V, 12, vol. 1, p. 63. Au sujet de la génération éternelle de Dieu, voir P. Deghaye, La naissance de Dieu, ou la doctrine de Jacob Boehme, Paris, Albin Michel, 1985.

57. Cf. De electione gratice (1623), I, 21. 
de son existence. Animé par le désir d'aller au-delà de ses limites, «l'homme est en devenir ${ }^{58}$.

Alors que les béguines du Moyen Âge invitaient l'âme à perdre son nom ${ }^{59}$ dans l'essence divine, Boehme encourage l'homme à devenir un «autre», à revivifier l'image céleste afin d'incarner le Christ en terre. L'âme ne doit pas s'anéantir, mais se réaliser; elle ne doit pas abandonner les limites de sa personnalité, mais revêtir le corps divin dans ce monde.

Selon Boehme on renaît dans la chair, et la personne nouvelle qui va surgir au cours de cette renaissance spirituelle est l'homme intérieur ( «notre homme secret $»^{60}$ ) qui s'est substitué à l'homme extérieur. La régénération dans le Christ est donc un procès de transformation personnelle accomplie dans la foi, dont la visée est de faire revivre l'âme en Dieu et non de disparaître pour être englouti dans le gouffre insondable d'une divinité abstraite. La mystique du théosophe de Görlitz n'est pas une mystique fusionnelle, mais une mystique de la rencontre entre une âme régénérée qui acquiert une nouvelle identité et un Dieu vivant qui remplit le lieu de ce monde ainsi que l'espace spirituel. Enfin l'âme renaît dans l'Autre, en Dieu, qui est partout et nulle part:

Quand elle cheminerait l'espace de mille milles, elle serait néanmoins dans le point d'où elle serait partie, car dans Dieu il n'y a aucune limite. Près et loin ne sont qu'une même chose. Elle est aussi rapide qu'une pensée des hommes. Elle est magique. Elle demeure dans ses merveilles. C'est là son habitation ${ }^{61}$.

Et dans ces espaces infinis l'âme découvre la liberté de l'Origine, car «celui qui est né au fond de son âme, engendré par le Verbe céleste, se meut librement dans l'éternité ${ }^{62}$.

58. Gerhard Wehr, Jakob Böhme in Selbstzeugnissen und Bilddokumenten, Hamburg, Rowohlt, 1971 (trad. fr. P. Kessler, in Jacob Böhme, Cahiers de l'Hermétisme, Paris, Albin Michel, 1977, p. 78).

59. Voir Marguerite Porete, Le miroir des simples âmes anéanties, Grenoble, J. Millon, 1991, p. 88 et 171.

60. J. Boehme, De triplici vita (1620); trad. fr. L.-C. de Saint-Martin, De la Triple vie de l'homme, selon le mystère des trois principes de la manifestation divine, Paris, Migneret, 1809, VI, 30.

61. Quarante questions, XXI, 4, p. 176; et quelques lignes plus haut: «Là où elle se trouve elle est dans le monde (abissal) insondable, où il n'y a ni fin ni limite dans lesquelles elle doive aller.»

62. De la signature des choses, XV, 24, p. 304. 
Accablé par l'hostilité de ses adversaires - notamment du pasteur luthérien Gregorius Richter - qui n'avaient pas compris la portée de ses révélations, malgré la censure, l'exil, et toutes les épreuves qu'il avait dû surmonter, le cordonnier mystique de Görlitz demeura néanmoins convaincu que l'homme est en mesure de pouvoir se régénérer et montrer Dieu; car l'homme d'après Boehme «est un buisson hérissé d'épines meurtrières. Mais parmi ces épines une rose fleurira et elle révélera le trésor caché ${ }^{63}$.

mariel.mazzocco@unige.ch

63. Ibid., ch. VIII, 52, p. 157. 